\title{
A SHORT PROOF OF A RESULT OF KATZ AND WEST
}

\author{
DIPANKAR GHOSH AND TONY J. PUTHENPURAKAL
}

\begin{abstract}
We give a short proof of a result due to Katz and West: Let $R$ be a Noetherian ring and $I_{1}, \ldots, I_{t}$ ideals of $R$. Let $M$ and $N$ be finitely generated $R$-modules and $N^{\prime} \subseteq N$ a submodule. For every fixed $i \geqslant 0$, the sets $\operatorname{Ass}_{R}\left(\operatorname{Ext}_{R}^{i}\left(M, N / I_{1}^{n_{1}} \cdots \bar{I}_{t}^{n_{t}} N^{\prime}\right)\right)$ and $\operatorname{Ass}_{R}\left(\operatorname{Tor}_{i}^{R}\left(M, N / I_{1}^{n_{1}} \cdots I_{t}^{n_{t}} N^{\prime}\right)\right)$ are independent of $\left(n_{1}, \ldots, n_{t}\right)$ for all sufficiently large $n_{1}, \ldots, n_{t}$.
\end{abstract}

Often in mathematics, once an interesting result has been established, other proof of the same result appears. In this article, we give a short proof of a result due to Katz and West.

Let $R$ be a commutative Noetherian ring with identity. Let $I$ be an ideal of $R$ and $M$ a finitely generated $R$-module. In [6], Ratliff conjectured about the asymptotic behaviour of the set of associated prime ideals $\operatorname{Ass}_{R}\left(R / I^{n}\right)$ (when $R$ is a domain). Subsequently, Brodmann [1] proved that $\operatorname{Ass}_{R}\left(M / I^{n} M\right)$ stabilizes for $n$ sufficiently large. Thereafter, this result was extended to an arbitrary finite collection of ideals by Kingsbury and Sharp in [4, Theorem 1.5]; see also [2, Corollary 1.8(c)].

In a different direction, Melkersson and Schenzel generalized Brodmann's result by showing that $\operatorname{Ass}_{R}\left(\operatorname{Tor}_{i}^{R}\left(M, R / I^{n}\right)\right)$ is independent of $n$ for all large $n$ and for every fixed $i \geqslant 0$; see [5. Theorem 1]. Recently, in [3, Corollary 3.5], Katz and West proved all the above results in a more general form:

Set-up 1. Let $I_{1}, \ldots, I_{t}$ be ideals of $R$. Suppose $M$ and $N$ are finitely generated $R$-modules and $N^{\prime} \subseteq N$ a submodule. We set $\mathbb{N}:=\{n \in \mathbb{Z}: n \geqslant 0\}$. Fix $i \in \mathbb{N}$. For every $\mathbf{n}:=\left(n_{1}, \ldots, n_{t}\right) \in \mathbb{N}^{t}$, we denote $\mathbf{I}^{\mathbf{n}}:=I_{1}^{n_{1}} \cdots I_{t}^{n_{t}}$ and we set

$$
W_{\mathbf{n}}:=\operatorname{Ext}_{R}^{i}\left(M, N / \mathbf{I}^{\mathbf{n}} N^{\prime}\right) \quad \text { and } \quad W_{\mathbf{n}}^{\prime}:=\operatorname{Tor}_{i}^{R}\left(M, N / \mathbf{I}^{\mathbf{n}} N^{\prime}\right) .
$$

Let $W:=\bigoplus_{\mathbf{n} \in \mathbb{N}^{t}} W_{\mathbf{n}}$ and $W^{\prime}:=\bigoplus_{\mathbf{n} \in \mathbb{N}^{t}} W_{\mathbf{n}}^{\prime}$.

With this set-up, Katz and West showed that $\operatorname{Ass}_{R}\left(W_{\mathbf{n}}\right)$ and $\operatorname{Ass}_{R}\left(W_{\mathbf{n}}^{\prime}\right)$ are independent of $\mathbf{n}$ for all $\mathbf{n} \gg 0$. The aim of this article is to give a short proof of this result. We prove all the results here for Ext-modules only. For the analogous result of Tor-modules, the proof goes through exactly the same way.

Discussion 2. For every $1 \leqslant j \leqslant t, \mathbf{e}^{j}$ denotes the $j$ th standard basis element of $\mathbb{N}^{t}$. Let $\mathscr{R}(\mathbf{I}):=\bigoplus_{\mathbf{n} \in \mathbb{N}^{t}} \mathbf{I}^{\mathbf{n}}$ be the $\mathbb{N}^{t}$-graded Rees ring. The short exact sequence

$$
0 \rightarrow \bigoplus_{\mathbf{n} \in \mathbb{N}^{t}} \mathbf{I}^{\mathbf{n}} N^{\prime} / \mathbf{I}^{\mathbf{n}+\mathbf{e}^{j}} N^{\prime} \longrightarrow \bigoplus_{\mathbf{n} \in \mathbb{N}^{t}} N / \mathbf{I}^{\mathbf{n}+\mathbf{e}^{j}} N^{\prime} \longrightarrow \bigoplus_{\mathbf{n} \in \mathbb{N}^{t}} N / \mathbf{I}^{\mathbf{n}} N^{\prime} \rightarrow 0
$$

yields the following exact sequence of $\mathbb{N}^{t}$-graded $\mathscr{R}(\mathbf{I})$-modules:

$$
\bigoplus_{\mathbf{n} \in \mathbb{N}^{t}} \operatorname{Ext}_{R}^{i}\left(M, \frac{\mathbf{I}^{\mathbf{n}} N^{\prime}}{\mathbf{I}^{\mathbf{n}+\mathbf{e}^{j}} N^{\prime}}\right) \stackrel{\Phi_{j}}{\longrightarrow} W\left(\mathbf{e}^{j}\right) \longrightarrow W \stackrel{\Psi_{j}}{\longrightarrow} \bigoplus_{\mathbf{n} \in \mathbb{N}^{t}} \operatorname{Ext}_{R}^{i+1}\left(M, \frac{\mathbf{I}^{\mathbf{n}} N^{\prime}}{\mathbf{I}^{\mathbf{n}+\mathbf{e}^{j}} N^{\prime}}\right),
$$

Date: February 5, 2016.

2010 Mathematics Subject Classification. Primary 13E05, 13D07; Secondary 13A15, 13A30.

Key words and phrases. Associate primes; Rees ring; Ext; Tor. 
where $W\left(\mathbf{e}^{j}\right)_{\mathbf{n}}:=W_{\mathbf{n}+\mathbf{e}^{j}}$ for all $\mathbf{n} \in \mathbb{N}^{t}$. Setting $U^{j}:=\operatorname{Image}\left(\Phi_{j}\right)$ and $V^{j}:=$ Image $\left(\Psi_{j}\right)$, we obtain the following exact sequence of $\mathbb{N}^{t}$-graded $\mathscr{R}(\mathbf{I})$-modules:

$$
0 \longrightarrow U^{j} \longrightarrow W\left(\mathbf{e}^{j}\right) \longrightarrow W \longrightarrow V^{j} \longrightarrow 0
$$

where $U^{j}$ and $V^{j}$ are finitely generated $\mathbb{N}^{t}$-graded $\mathscr{R}(\mathbf{I})$-modules.

We say an $\mathbb{N}^{t}$-graded module $U$ is eventually zero (resp. non-zero) if $U_{\mathbf{n}}=0$ for all $\mathbf{n} \gg 0$ (resp. $U_{\mathbf{n}} \neq 0$ for all $\mathbf{n} \gg 0$ ). By virtue of [7, Proposition 5.1], a finitely generated $\mathbb{N}^{t}$-graded $\mathscr{R}(\mathbf{I})$-module is either eventually zero or eventually non-zero.

Lemma 3. With the Set-up 1, if $(R, \mathfrak{m}, k)$ is a local ring, then each of $\operatorname{Hom}_{R}(k, W)$ and $\operatorname{Hom}_{R}\left(k, W^{\prime}\right)$ is either eventually zero or eventually non-zero.

Proof. For every $1 \leqslant j \leqslant t$, in view of (2.1), by setting $X^{j}:=\operatorname{Image}\left(W\left(\mathbf{e}^{j}\right) \rightarrow W\right)$, we obtain the following short exact sequences of $\mathbb{N}^{t}$-graded $\mathscr{R}(\mathbf{I})$-modules:

$$
0 \rightarrow U^{j} \rightarrow W\left(\mathbf{e}^{j}\right) \rightarrow X^{j} \rightarrow 0 \text { and } 0 \rightarrow X^{j} \rightarrow W \rightarrow V^{j} \rightarrow 0,
$$

which induce the following exact sequences of $\mathbb{N}^{t}$-graded $\mathscr{R}(\mathbf{I})$-modules:

$$
\begin{aligned}
& 0 \rightarrow \operatorname{Hom}_{R}\left(k, U^{j}\right) \rightarrow \operatorname{Hom}_{R}\left(k, W\left(\mathbf{e}^{j}\right)\right) \rightarrow \operatorname{Hom}_{R}\left(k, X^{j}\right) \rightarrow Y^{j} \rightarrow 0, \\
& 0 \rightarrow \operatorname{Hom}_{R}\left(k, X^{j}\right) \rightarrow \operatorname{Hom}_{R}(k, W) \rightarrow Z^{j} \rightarrow 0,
\end{aligned}
$$

where $Y^{j}$ and $Z^{j}$ (being submodules of $\operatorname{Ext}_{R}^{1}\left(k, U^{j}\right)$ and $\operatorname{Hom}_{R}\left(k, V^{j}\right)$ respectively) are finitely generated $\mathbb{N}^{t}$-graded $\mathscr{R}(\mathbf{I})$-modules. It can be observed from (3.1) and (3.2) that if any one of $\operatorname{Hom}_{R}\left(k, U^{j}\right), Y^{j}$ and $Z^{j}(1 \leqslant j \leqslant t)$ is eventually non-zero, then so is $\operatorname{Hom}_{R}(k, W)$, and we are done. So we may assume that $\operatorname{Hom}_{R}\left(k, U^{j}\right)$, $Y^{j}$ and $Z^{j}$ are eventually zero for all $1 \leqslant j \leqslant t$. In this case, setting $f(\mathbf{n}):=$ length $\left(\operatorname{Hom}_{R}\left(k, W_{\mathbf{n}}\right)\right)$ for all $\mathbf{n} \in \mathbb{N}^{t}$, in view of the $\mathbf{n}$ th components of (3.1) and (3.2), we obtain that $f\left(\mathbf{n}+\mathbf{e}^{j}\right)=f(\mathbf{n})$ for all $1 \leqslant j \leqslant t$ and for all $\mathbf{n} \gg 0$. Therefore $f(\mathbf{n})=c$ for all $\mathbf{n} \gg 0$, where $c$ is a constant. The lemma now follows easily.

Now we can achieve the aim of this article.

Theorem 4. With the Set-up 1, there exists $\mathbf{k} \in \mathbb{N}^{t}$ such that the sets $\operatorname{Ass}_{R}\left(W_{\mathbf{n}}\right)$ and $\operatorname{Ass}_{R}\left(W_{\mathbf{n}}^{\prime}\right)$ are independent of $\mathbf{n}$ for all $\mathbf{n} \geqslant \mathbf{k}$.

Proof. We first show that $\bigcup_{\mathbf{n} \in \mathbb{N}^{t}} \operatorname{Ass}_{R}\left(W_{\mathbf{n}}\right)$ is finite. For every $\mathbf{n} \in \mathbb{N}^{t}$, the $\mathbf{n}$ th component of the exact sequence (2.1) (for $j=1$ ) gives

$$
\begin{aligned}
\operatorname{Ass}_{R}\left(W_{\mathbf{n}+\mathbf{e}^{1}}\right) & \subseteq \operatorname{Ass}_{R}\left(U_{\mathbf{n}}^{1}\right) \cup \operatorname{Ass}_{R}\left(W_{\mathbf{n}}\right) \\
& \subseteq \operatorname{Ass}_{R}\left(U_{\mathbf{n}}^{1}\right) \cup \operatorname{Ass}_{R}\left(U_{\mathbf{n}-\mathbf{e}^{1}}^{1}\right) \cup \operatorname{Ass}_{A}\left(W_{\mathbf{n}-\mathbf{e}^{1}}\right) \\
& \cdots \\
& \subseteq\left(\bigcup_{0 \leqslant l \leqslant n_{1}} \operatorname{Ass}_{R}\left(U_{\left(l, n_{2}, \ldots, n_{t}\right)}^{1}\right)\right) \bigcup \operatorname{Ass}_{R}\left(W_{\left(0, n_{2}, \ldots, n_{t}\right)}\right) .
\end{aligned}
$$

Taking union over $\mathbf{n} \in \mathbb{N}^{t}$, we obtain that

$$
\bigcup_{\mathbf{n} \in \mathbb{N}^{t}} \operatorname{Ass}_{R}\left(W_{\mathbf{n}}\right) \subseteq\left(\bigcup_{\mathbf{n} \in \mathbb{N}^{t}} \operatorname{Ass}_{R}\left(U_{\mathbf{n}}^{1}\right)\right) \bigcup\left(\bigcup_{\left(n_{2}, \ldots, n_{t}\right) \in \mathbb{N}^{t-1}} \operatorname{Ass}_{R}\left(W_{\left(0, n_{2}, \ldots, n_{t}\right)}\right)\right) .
$$

Since $U^{1}$ is finitely generated, the set $\bigcup_{\mathbf{n} \in \mathbb{N}^{t}} \operatorname{Ass}_{R}\left(U_{\mathbf{n}}^{1}\right)$ is finite; see [7, Lemma 3.2]. Therefore one obtains that $\bigcup_{\mathbf{n} \in \mathbb{N}^{t}} \operatorname{Ass}_{R}\left(W_{\mathbf{n}}\right)$ is finite by using induction on $t$.

Since $\bigcup_{\mathbf{n} \in \mathbb{N}^{t}} \operatorname{Ass}_{R}\left(W_{\mathbf{n}}\right)$ is finite, it is now enough to prove that for every $\mathfrak{p} \in$ $\bigcup_{\mathbf{n} \in \mathbb{N}^{t}} \operatorname{Ass}_{R}\left(W_{\mathbf{n}}\right)$, exactly one of the following alternatives must hold: either $\mathfrak{p} \in$ 
$\operatorname{Ass}_{R}\left(W_{\mathbf{n}}\right)$ for all $\mathbf{n} \gg 0$; or $\mathfrak{p} \notin \operatorname{Ass}_{R}\left(W_{\mathbf{n}}\right)$ for all $\mathbf{n} \gg 0$. Localizing at $\mathfrak{p}$, and replacing $R_{\mathfrak{p}}$ by $R$ and $\mathfrak{p} R_{\mathfrak{p}}$ by $\mathfrak{m}$, it is now enough to prove that either $\mathfrak{m} \in$ $\operatorname{Ass}_{R}\left(W_{\mathbf{n}}\right)$ for all $\mathbf{n} \gg 0$; or $\mathfrak{m} \notin \operatorname{Ass}_{R}\left(W_{\mathbf{n}}\right)$ for all $\mathbf{n} \gg 0$, which is equivalent to that either $\operatorname{Hom}_{R}\left(k, W_{\mathbf{n}}\right) \neq 0$ for all $\mathbf{n} \gg 0$; or $\operatorname{Hom}_{R}\left(k, W_{\mathbf{n}}\right)=0$ for all $\mathbf{n} \gg 0$, where $k:=R / \mathfrak{m}$. The last result follows from Lemma 3 .

\section{ACKNOWLEDGEMENTS}

The first author would like to thank NBHM, DAE, Govt. of India for providing financial support for this study.

\section{REFERENCES}

[1] M. Brodmann, Asymptotic stability of $\operatorname{Ass}\left(M / I^{n} M\right)$, Proc. Amer. Math. Soc. 74 (1979), $16-18$.

[2] D. Katz, S. McAdam and L. J. Ratliff, Jr., Prime divisors and divisorial ideals, J. Pure Appl. Algebra 59 (1989), 179-186.

[3] D. Katz and E. West. A linear function associated to asymptotic prime divisors. Proc. Amer. Math. Soc. (6) 132 (2004), 1589-1597.

[4] A. K. Kingsbury and R. Y. Sharp, Asymptotic behaviour of certain sets of prime ideals, Proc. Amer. Math. Soc. 124 (1996), 1703-1711.

[5] L. Melkersson and P. Schenzel, Asymptotic prime ideals related to derived functors, Proc. Amer. Math. Soc. 117 (1993), 935-938.

[6] L. J. Ratliff, Jr., On prime divisors of $I^{n}$, $n$ large, Michigan Math. J. 23 (1976), 337-352.

[7] E. West, Primes associated to multigraded modules, J. Algebra 271 (2004), 427-453.

Department of Mathematics, Indian Institute of Technology Bombay, Powai, Mumbai 400076, INDIA

E-mail address: dipankar@math.iitb.ac.in, dipug23@gmail.com (Dipankar Ghosh)

E-mail address: tputhen@math.iitb.ac.in (Tony J. Puthenpurakal) 